\title{
Meant to merge: Fabrication of stretchy electronics for robotics
}

\author{
Florian Hartmann ${ }^{1}$, Michael Drack ${ }^{1}$, Martin Kaltenbrunner ${ }^{1 *}$ \\ ${ }^{1}$ Soft Electronics Laboratory, Linz Institute of Technology, Johannes Kepler University Linz, \\ Altenbergerstrasse 69, 4040 Linz, Austria. \\ *Corresponding author. Email: martin.kaltenbrunner@jku.at
}

In essence, we are sensor-clad soft machines capable of myriad intricate tasks. Stripped from proprioceptive feedback, we can no longer walk despite intact locomotor system. Likewise, integration of sensors, complex control loops, or machine learning is crucial in "classical" robotics. Soft and continuum robots mitigate some materials and engineering issues when trying to replicate biological systems (1) through compliant and adaptive structures that allow impressive functionality with limited or no sensing $(2,3)$. The initial charm of simplicity lowered the barrier of entry to soft robotics and partly sparked its rapid growth (4). Yet, in nature's soft experts, such as cephalopods, we find intricate networks of sensors, actuators, power supply, and signal processing distributed throughout their squishy bodies. Many current soft actuator demonstrations partially compensate through their operator's knowledge of the environment or the desired task. Truly autonomous robots, soft or rigid, require a sense of their own to interact with their surroundings. How can we best approximate the inimitable complexity of living beings in our artificial critters? This endeavor can be successful only if material scientists, chemists, engineers, physicists, biologists, and roboticists join forces. In particular, soft embodiments in robotics necessitate stretchable, deformable forms of electronics - for sensing, power supply, computing, and actuation support-that do not impede their inherently compliant and safe nature (Fig. 1). Immersing robots in our daily lives demands low production costs for soft sensor skins. Scalable solutions of flexible electronics for stretchable systems are being adopted; demonstrations of embedded soft sensors manufactured via digital fabrication are encouraging. Monolithic assembly of multiple materials on large scales with high functionality yet in a robust manner, while achieving untethered operation, requires new integration paradigms.

\section{Grand challenges}

To avoid negating the advantages of soft machines that originate in mechanical design concepts and materials choices that off-load some control tasks to the soft structure [morphological computation (4)], we first need to ask what a soft robot needs to sense. Evolutionarily, the realization of sensors is driven by environmental needs. In analogy, we should equip our creations with just the right amount of responsiveness and take advantage of self-sensing actuation principles.

\section{Stay simple in a frugal way}

Electrically driven soft capacitive devices not only provide motion but also allow closed-loop control via the strain-dependent capacity change that correlates with the actuation state. Recent PeanoHASEL actuators couple the fine control of electric operation with the high power of fluid-based systems in next-generation artificial muscles (5). They overcome dielectric breakdown failure partly through fluid redistribution, but advances in self-healable polymers, elastomers, and electrode materials could further boost longevity. Being electronic in nature, an evolution toward multimodal sensing is promising. Existing strategies for soft sensing include resistive- and capacitive-based designs to perceive pressure and strain, with attempts toward light-based feedback (6). Going 
beyond smarter actuators toward "sentient" robots will require a broad spectrum of senses, realized in soft form factors, spanning temperature, moisture, flow, texture, optics, and electromagnetic fields. Materials that respond to multiple stimuli may simplify sensor design and skin architecture. However, integration strategies for sensing, data collection and processing, and energy storage are paramount.

\section{Collecting a lot of signals}

Whenever a single or a small number of sensors do not suffice, addressing individual nodes quickly becomes a major obstacle. Approaches from the display industry, where millions of pixels must be controlled, use active matrix transistor arrays that require heterogeneous integration of different materials classes-dielectrics, semiconductors, and metals-all with partially antagonistic properties. Examples that use organic (7) and inorganic (8) semiconductors as essential building blocks enabled epidermal and imperceptible foil-based platforms with exceptional mechanical compliance through their ultrathin form factors. Mechanical designs such as meanders, cracked metal films, or out-ofplane wrinkles then turn flexible e-skins into stretchable soft hybrids when combined with elastic foundations. Advances in intrinsically stretchable (semi)conductors and insulators now provide allsoft and printable alternatives (9). Long-term reliability remains challenging; redundancy in sensors and materials with basic healing properties may offer solutions.

\section{Processing and adaptation}

Stretchable sensors benefit from the intrinsic nonlinearities of their constituting materials, but signal interpretation is challenging and typically changes over time. Advanced processing and communication largely rely on rigid electronic components; interfacing with stretchable materials involves soft-to-hard transitions that risk failure when undergoing large cyclic stresses. Self-healing materials with graded mechanical modulus could help; another strategy may lie in modular designs where the sensor skin can be separated and replaced. Advances in machine learning can be explored via "tethered learning" even in small, autonomous robots. Linked to large resources while empirically training their soft senses, these robots then locally store created patterns to guide evaluations of sensor inputs, allowing adaptations to the environment at lower computational and thus energy demand.

\section{Providing energy}

Distributed energy harvesting from low-density sources (e.g., vibrations and thermal gradients) is promising to power individual sensors. Even self-powered sensors are possible by choosing piezo-, tribo-, or photoelectric active materials. But if we look for active display or shape-morphing skins, we require strategies to seamlessly integrate high-density sources using, for example, electro-chemical or efficient solar converters that have dual purpose and can also power locomotion. Existing stretchable versions suffer from long-term stability issues. Encapsulation and barrier materials that stretch are elusive but may be possible with engineered two-dimensional materials. Tapping the advanced power management of biological systems via incorporating living cells for actuation (10) and sensing could provide a major step forward for autonomous systems that ultimately heal and regenerate naturally. Yet, this ability poses new challenges in supplying nutrition and handling waste, especially for large systems. Hydrogel-based electronics could offer a natural solution.

\section{Where to go}

A growing complexity and number of sensors in turn demand appropriate power supply and computational resources. Distributing not only signal processing but also energy storage throughout the robotic body is closely analogous to biological systems and provides a realistic solution for 
practical implementations. Soon, a synergistic concept that harnesses the transformative achievements of silicon technology in conjunction with large-area e-skins through hybrid integration strategies will be an intermediate on our way toward all-soft systems. Next-level soft robotics will include emerging concepts for self-healing the body and the somatosensory skins. Because unlimited operational lifetime is neither feasible nor required, the final touch that blurs the lines between digital and animate worlds will be provided through biodegradable and resorbable materials and designs.

\section{Acknowledgments}

We are indebted to S. Bauer for stimulating discussions. Funding: This work was supported by the European Research Council Starting Grant "GEL-SYS" (grant agreement no. 757931) and a startup grant of the Linz Institute of Technology (grant agreement no. LIT013144001SEL).

\section{References and notes}

1. C. Laschi, M. Cianchetti, B. Mazzolai, L. Margheri, M. Follador, P. Dario, Soft robot arm inspired by the octopus. Adv. Robotics 26, 709-727 (2012).

2. K. Suzumori, S. likura, H. Tanaka, Development of flexible microactuator and its applications to robotic mechanisms, Proceedings of the 1991 IEEE International Conference on Robotics and Automation, Sacramento, CA, 9 to 11 April 1991, pp. 1622-1627

3. R. F. Shepherd, F. Ilievski, W. Choi, S. A. Morin, A. A. Stokes, A. D. Mazzeo, X. Chen, M. Wang, G. M. Whitesides, Multigait soft robot. Proc. Natl. Acad. Sci. U.S.A. 108, 20400-20403 (2011).

4. Y. Mengüç, N. Correll, R. Kramer, J. Paik, Will robots be bodies with brains or brains with bodies? Sci. Robot. 2, eaar4527 (2017).

5. N. Kellaris, V. G. Venkata, G. M. Smith, S. K. Mitchell, C. Keplinger, Peano-HASEL actuators: Musclemimetic, electrohydraulic transducers that linearly contract on activation. Sci. Robot. 3, eaar3276 (2018).

6. Towards soft perceptive robots: From robotics and biologically-inspired solutions to soft sensing technologies, Workshop at the IEEE RoboSoft Conference, Livorno, Italy, 24 to 28 April 2018; https://softperceptiverobots.it/

7. M. Kaltenbrunner, T. Sekitani, J. Reeder, T. Yokota, K. Kuribara, T. Tokuhara, M. Drack, R. Schwödiauer, I. Graz, S. Bauer-Gogonea, S. Bauer, T. Someya, An ultra-lightweight design for imperceptible plastic electronics. Nature 499, 458-463 (2013).

8. D.-H. Kim, N. Lu, R. Ma, Y.-S. Kim, R.-H. Kim, S. Wang, J. Wu, S. M. Won, H. Tao, A. Islam, K. J. Yu, T.i. Kim, R. Chowdhury, M. Ying, L. Xu, M. Li, H.-J. Chung, H. Keum, M. McCormick, P. Liu, Y.-W. Zhang, F. G. Omenetto, Y. Huang, T. Coleman, J. A. Rogers, Epidermal electronics. Science 333, 838-843 (2011).

9. S. Wang, J. Xu, W. Wang, G.-J. N. Wang, R. Rastak, F. Molina-Lopez, J. W. Chung, S. Niu, V. R. Feig, J. Lopez, T. Lei, S.-K. Kwon, Y. Kim, A. M. Foudeh, A. Ehrlich, A. Gasperini, Y. Yun, B. Murmann, J. B.-H. Tok, Z. Bao, Skin electronics from scalable fabrication of an intrinsically stretchable transistor array. Nature 555, 83-88 (2018).

10. L. Ricotti, B. Trimmer, A. W. Feinberg, R. Raman, K. K. Parker, R. Bashir, M. Sitti, S. Martel, P. Dario, A. Menciassi, Biohybrid actuators for robotics: A review of devices actuated by living cells. Sci. Robot. 2, eaaq0495 (2017). 


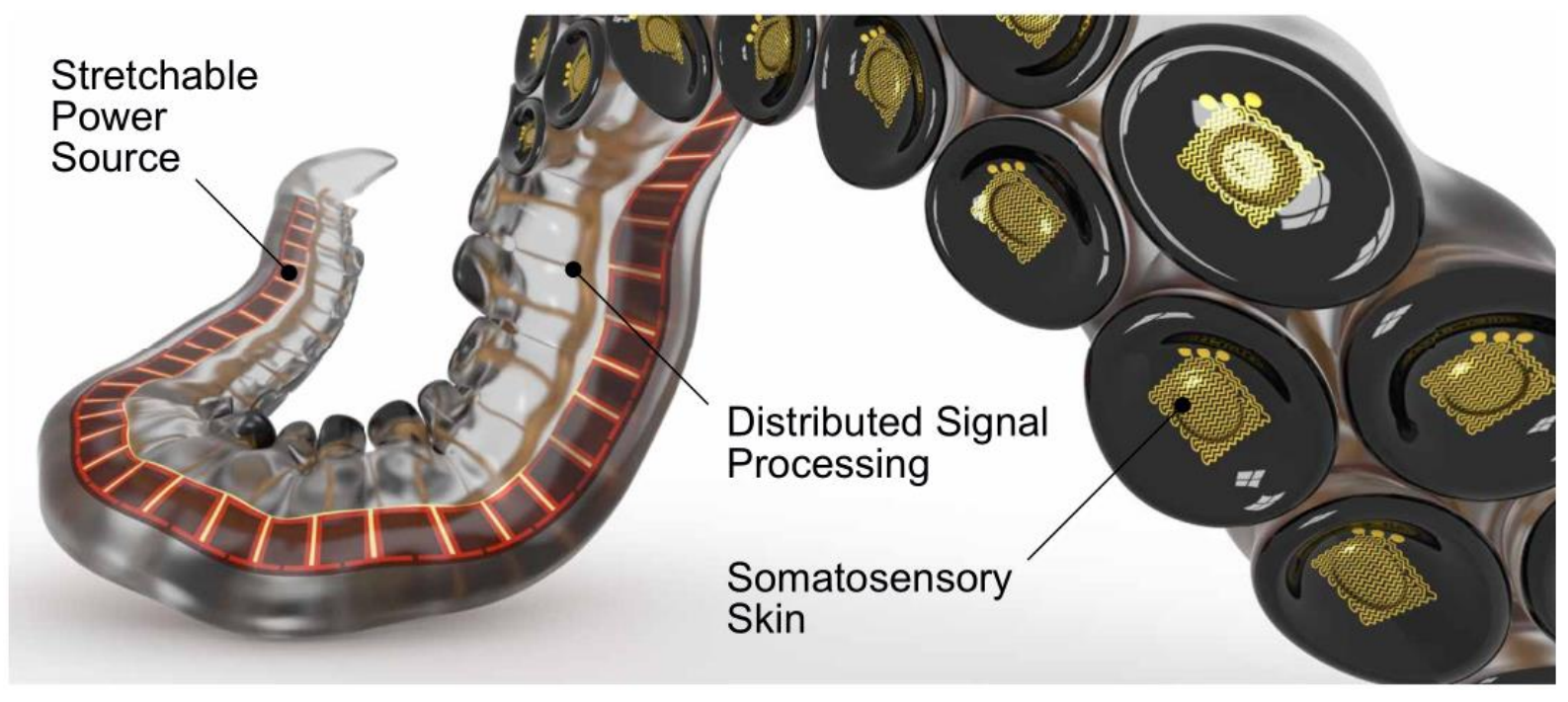

Fig. 1. Narrowing the gap between biological and robotic systems. All-soft robots ideally integrate soft actuation with stretchable, multimodal electronic skins and energy supply. Untethered autonomous operation is the ultimate goal. 